\title{
Near-Field Cosmology with RR Lyrae Variable Stars: A First View of Substructure in the Southern Sky
}

\author{
C. Navarrete ${ }^{1,2}$, S. Duffau ${ }^{2,1}$, A. K. Vivas ${ }^{3}$, M. Catelan ${ }^{1,2}$, \\ G. Hajdu ${ }^{1,2}$, G. Torrealba ${ }^{4}$, C. Cortés ${ }^{5,2}$, V. Belokurov ${ }^{4}$, \\ S. Koposov ${ }^{4}$ and A. J. Drake ${ }^{6}$ \\ ${ }^{1}$ Instituto de Astrofísica, Pontificia Universidad Católica de Chile, \\ Av. Vicuña Mackenna 4860, 782-0436 Macul, Santiago, Chile \\ email: [cnavarre, sduffau] @astro.puc.cl \\ ${ }^{2}$ Millennium Institute of Astrophysics, Santiago, Chile \\ ${ }^{3}$ Cerro Tololo Inter-American Observatory, Casilla 603, La Serena, Chile \\ ${ }^{4}$ Institute of Astronomy, Madingley Road, Cambridge CB3 0HA, UK \\ ${ }^{5}$ Departamento de Física, Facultad de Ciencias Básicas, Universidad Metropolitana de \\ Ciencias de la Educación, Av. José Pedro Alessandri 774, 776-0197 Ñuñoa, Santiago, Chile \\ ${ }^{6}$ California Institute of Technology, 1200 East California Boulevard, Pasadena, CA 91225, USA

\begin{abstract}
We present the current status of the spectroscopic follow-up of a large number of RR Lyrae (RRL) halo overdensity candidates recently found by Torrealba et al. (2015) using southern-hemisphere data from the Catalina Real-time Transient Survey (CRTS). Characterizing the individual RRL stars in these overdensities is crucial to confirm them as real halo substructures. Low-resolution spectra have been obtained for RRL stars in 11 different overdensities, using the SOAR and Magellan telescopes. Radial velocities and metallicities have been derived so far for 123 and 99 RRL stars, respectively.
\end{abstract}

Keywords. Galaxy: halo, Galaxy: structure, stars: variables: RR Lyrae

A complete census of substructure in the Milky Way halo is essential to understand the history of formation of our Galaxy, and thereby provide constraints on models of hierarchical galaxy formation. Until recently, however, the southern celestial hemisphere has remained virtually uncharted territory, with halo substructure having mostly been identified in the north. The situation is now beginning to change; in particular, Torrealba et al. (2015, hereinafter T15) have recently provided a list of southern overdensity candidates based on RRL stars identified using CRTS data. Spectroscopic follow-up of the individual RRL stars in these overdensity candidates is now required to confirm their true nature, or to discard them as artifacts or random clumps among halo field stars.

The main goal of our project is to perform spectroscopic follow-up of the T15 candidates, thereby deriving radial velocities $(\mathrm{RVs})$ and metallicities $([\mathrm{Fe} / \mathrm{H}])$ of individual RRL contained in these overdensities. This, together with their distances and other information from the literature (when available), will help us to properly establish the physical properties of each overdensity. By comparing with data for other previously known substructures, including those from the northern hemisphere, we also hope to establish any possible links that may exist amongst them.

Low-resolution $(R \sim 2000)$ spectra have been obtained using LDSS3@LCO and Goodman@SOAR for $\approx 200$ stars covering 11 of the 27 overdensities from the T15 catalog, including Sgr 1, Crv 1, Hya 1, and Hya 2. Figure 1 displays the RRL from T15 and those 


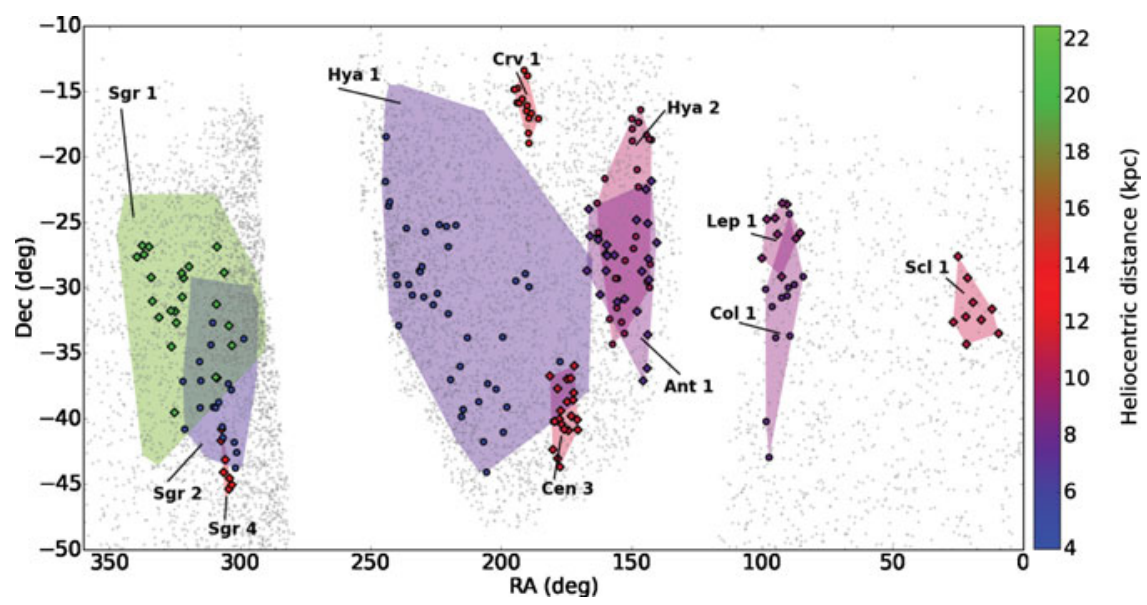

Figure 1. Spatial distribution of the targeted RRL overdensities (shaded polygons). Filled symbols correspond to RRLs with spectra while gray points are all the RRLs in the T15 catalog. Heliocentric distances are color-coded according to the scale on the right.

observed by us in each of the targeted overdensities. RVs have been measured for 123 stars, and corrected for their pulsation phase using the Sesar (2012) templates. [Fe/H] has been estimated for 99 stars using the method of Layden (1994). Typical errors are $\sim 20 \mathrm{~km} \mathrm{~s}^{-1}$ and $0.2 \mathrm{dex}$, respectively. Our preliminary analysis tentatively suggests a connection between Crv 1 and the Virgo Stellar Stream (Duffau et al. 2006), and reveals an interesting bimodal radial velocity distribution for Hya 2. Hya 1 is of special interest since Casetti-Dinescu et al. (2015) identified a moving group in this part of the sky. Our RVs together with proper motions will allow us to study in detail the nature of this object. Testing of the relation between Sgr 1 and the Sagittarius tidal stream using both data (e.g., Koposov et al. 2012) and disruption models (e.g., Law \& Majewski 2010) will also be afforded by our data. Detailed comparisons with models of the smooth halo will be carried out to help confirm or reject these connections, and to assess their significance.

\section{Acknowledgements}

This project is supported by CONICYT through International Research Project DPI 20140066. Additional support is provided by the Ministry for the Economy, Development, and Tourism's Iniciativa Científica Milenio through grant IC 120009, awarded to the Millennium Institute of Astrophysics; by Proyecto Fondecyt Regular \#1141141; and by Proyecto Basal PFB-06/2007. C.N. and G.H. gratefully acknowledge support from CONICYT-PCHA/Doctorado Nacional grants 2015-21151643 and 2014-63140099, respectively. C.N. also acknowledges additional support from a SOCHIAS grant through Gemini/CONICYT Project \#32140015.

\section{References}

Casetti-Dinescu, D. I., Nusdeo, D. A., Girard, T. M., et al. 2015, ApJ, 810, 4

Duffau, S., Zinn, R., Vivas, A. K., et al. 2006, ApJ, 636, 97

Law, D. R. \& Majewski, S. R. 2010, ApJ, 714, 229

Layden, A. C. 1994, AJ, 108, 1016

Koposov, S. E., Belokurov, V., Evans, N. W., et al. 2012, ApJ, 750, 80

Sesar, B. 2012, AJ, 144, 114

Torrealba, G., Catelan, M., Drake, A. J., et al. 2015, MNRAS, 446, 2251 